\title{
Modelling of metal-to-metal seals in a pressure relief valve using advanced $\mathrm{FE}$-analysis
}

\author{
Y. Gorash, W. Dempster, W. D. Nicholls \& R. Hamilton \\ Department of Mechanical and Aerospace Engineering, \\ University of Strathclyde, UK
}

\begin{abstract}
This study investigates the behaviour of the contact faces in the metal-to-metal seal of a typical pressure relief valve. The valve geometry is simplified to an axisymmetric problem. A cylindrical nozzle, which has a valve seat on top, contacts with a disk, which is preloaded by a compressed linear spring. All the components are made of the steel AISI type $316 \mathrm{~N}(\mathrm{~L})$ defined using the multilinear kinematic hardening material model based on monotonic and cyclic tests at $20^{\circ} \mathrm{C}$. Analysis considerations include the effects of the Fluid Pressure Penetration (FPP) across the valve seat which exists at two different scales. There is certain limited fluid leakage through the valve seat at operational pressures, which is caused by the fluid penetrating into surface asperities at the microscale. At the macroscale, nonlinear FE-analysis using the FPP technique available in ANSYS revealed that there is also a limited amount of fluid penetrating into gap. Accurate prediction of the fluid pressure profile over the valve seat is addressed in this study by considering the FPP interaction on both scales. The shape of this pressure profile introduces an additional component of the spring force, which needs to be considered to provide a reliable sealing. The analysis showed that the evolution of the profile, which is caused by the isotropic softening of the material, is significant during the cyclic operation of the valve.
\end{abstract}

Keywords: contact, finite element analysis, metal-to-metal seal, plasticity, pressure penetration, safety valve, type 316 steel.

\section{Introduction}

Static sealing is a fluid-structure coupled problem where the degree of leak tightness is dictated by the local and global deformation of the contact surfaces. 
The leakage paths result from gaps at the contact faces and are at a geometrical scale of the surface asperities which are at the microns scale. The contact face global geometry is at a macro scale where its resulting deformation is due to the global force loading, which in the case of a safety valve is determined by the spring forces and the operating pressure conditions. This coupling between fluid and structure at geometric scales that range from the micro to the macro imposes considerable challenges to the analysis of the problem.

This paper presents the results of a study that is envisaged to investigate the development of computational analysis methods for the design of static metalto-metal seals in conventional spring loaded safety valves. The investigation is limited to global deformation at a macro scale with some initial simplified coupling from the micro to macro scale via pressure penetration and an imposed pressure variation across the sealing face.

\subsection{Background}

Pressure relief valves (PRVs), as discussed by Malek [1], are commonly used as a safety device in industrial processes to provide a self-regulating pressure release. The PRV is a type of valve used to control or limit the pressure in a system or vessel, which can build up by a process upset, instrument or equipment failure, or fire. The pressure is relieved by allowing the pressurised fluid to flow through the valve orifice out of the system.

It was observed by Song et al. [2] that in actual usage PRVs can sometimes start to release fluid prior to their set pressures $P_{\text {set }}$. This is particularly true when $P_{\text {set }}$ is defined as the opening pressure and can result in a degradation of leak tightness for operation pressure $P_{\mathrm{op}}$, which is typically $90 \%$ of $P_{\text {set }}$ referring to [3]. This study models the structure of the valve seat-disc interface with the aim of simulating the fluid pressure penetration effects. An advanced FEA is implemented in order to investigate and quantify the influence of these effects on a spring force, which is required to provide a reliable leak tightness for pressures below $P_{\text {set. }}$.

This study focusses on spring-operated metal-seated PRVs since they are not limited in temperature and pressure, when compared to the soft-seated PRVs, which have much more preferable leak tightness [3]. This range of valves often operate at high temperatures $\left(>300^{\circ} \mathrm{C}\right)$ which exclude the use of discs with soft seals, meaning that metal-to-metal contacts between the nozzle and disc are required to form the basis of fluid sealing. Fluid leakage is a major concern for metal-to-metal contacts across the entire range of operation, not just as the system pressure tends towards $P_{\text {set }}$.

\subsection{Concept of contact behaviour}

An idealised functional model of the PRV consists of the following three basic components, as shown in Fig. 1:

- Cylindrical nozzle defined by the radius of orifice $R_{\text {orif }}$ and the length of valve seat $L_{\text {seat }}$. 

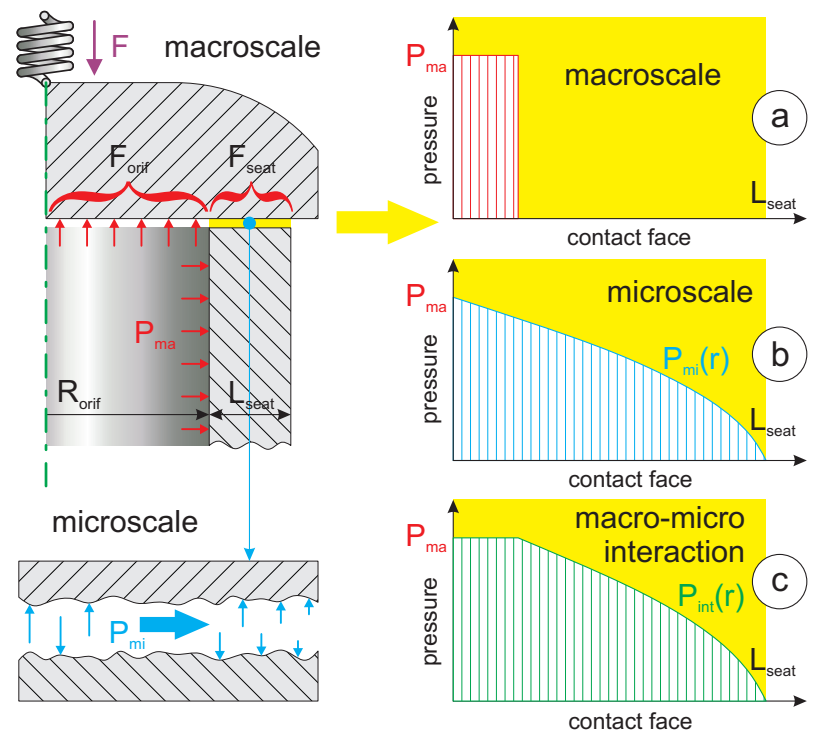

Figure 1: Concept of macro-micro effects interaction in the contact area of metalto-metal seal of a PRV.

- Relatively rigid disc, which keeps the nozzle closed during the normal operation of the valve.

- Linear longitudinal spring, which is initially compressed and prevents lifting of the disc during normal operation.

The degree of spring compression is adjusted to fit the set pressure $P_{\text {set }}$. So the spring force is equal to the force produced by the internal pressure, when it reaches the value of $P_{\text {set }}$, and applied to the disc surface corresponding to the orifice area. Since the system pressure $P_{\mathrm{ma}}$ is below $P_{\text {set }}$ during the normal operation, the orifice is kept tightly closed by the disc, providing a reliable seal through the difference of forces applied to the disc. When the pressure builds up, a weak balance of forces is achieved. Even a slight excess of $P_{\text {set }}$ starts the disk lifting. This operation description is true only for an idealised (perfectly elastic) material of the PRV. Since the real material, which is used for nozzle-disc pair, is quite far from perfectly elastic, the following concept has been proposed and shown in Fig. 1.

An internal edge of the valve seat is subjected to significant plastic deformation on the microscale, which is caused by the non-uniform contact conditions over the contact face. Once the valve is subjected to operational conditions, the pressurised fluid penetrates into the contact gap in the deformed contact face, as shown schematically on diagram in Fig. 1a. On the other hand, some limited degree of leakage is always practically observed in the metal-seated valves within the whole range of operation pressures. Since the contact is not perfectly tight, there is a fluid pressure penetration (FPP) in the valve seat over the whole contact face, 
as shown on diagram in Fig. 1b. Therefore, the FPP effects are observed at two different scales - macroscopic and microscopic. Since they both are assumed to exist in the same location, there should be an interaction between them. Prediction of the pressure profile over the contact face as a result of this interaction is a way to assess an additional component of the force produced by this pressure profile. Thus, the spring force can be calculated more accurately as a sum of 'orifice' and 'seat' components. An advanced FE-analysis using ANSYS is implemented for the prediction of pressure profile as a result of macro-micro interaction as shown on diagram in Fig. 1c.

The proposed analysis concept is applied to an investigation of contact behaviour in a typical spring-operated PRV with a medium size orifice ' $J$ ' according to API Standard 526. An important fact is that the disc and nozzle are both made of the austenitic stainless steel AISI 316N(L). It should be noted that the length of contact face of the valve seat in this PRV is relatively small when compared to the diameter of orifice.

\section{Material characterisation and modelling}

Common use of the AISI type $316 \mathrm{~N}$ (L) steel is for superheater piping, pressure vessels, heat exchangers and other components exposed to high temperatures of $650^{\circ} \mathrm{C}$ or higher, as indicated in previous work [4]. Since all the dynamic effects of valve lifting and resetting are neglected, a PRV operation is assumed to be quasistatic for FE-simulation in ANSYS. Therefore, the viscoplastic material behaviour of the steel AISI type $316 \mathrm{~N}(\mathrm{~L})$ is simplified to rate-independent plasticity neglecting viscous effects. Among the variety of plasticity models, the following formulations are considered for the FEA based upon the available monotonic and cyclic experiments [5] at $20^{\circ} \mathrm{C}$ and high strain rate regime $\dot{\varepsilon}=$ $10^{-4}-10^{-3} \mathrm{~s}^{-1}$ shown in Fig. 2:

1. Elastic-perfectly-plastic (EPP) fit presented by isotropic model with nohardening and yield stress $\bar{\sigma}_{\mathrm{y}}$ in ANSYS.

2. Smooth Ramberg-Osgood (R-O) fit presented by multilinear kinematic hardening (MLKH) model in ANSYS.

3. Combined hardening/softening rate-independent Chaboche model.

Experimental data for AISI type $316 \mathrm{~N}(\mathrm{~L})$ steel at $20^{\circ} \mathrm{C}$ from [5] is presented by two sets of data points shown in Fig. 2. The first set is a monotonic tensile stress-strain curve (SSC) in coordinates $\left[\varepsilon^{\text {tot }} ; \sigma\right]$, while the second set is a result of many cyclic tests, which were performed at different constant $\Delta \varepsilon^{\text {tot }}$ in coordinates $\left[\Delta \varepsilon^{\text {tot }} / 2 ; \Delta \sigma / 2\right]$. AISI type $316 \mathrm{~N}(\mathrm{~L})$ steel demonstrates mixed hardening-softening cyclic behaviour, which was comprehensively studied and modelled by Nouailhas et al. [7].

Since both experimental SSCs in Fig. 2 demonstrate some level of scatter, the first step in data analysis for the material model formulation is curve fitting. The conventional Ramberg-Osgood (R-O) equation [8] is optimal for such curve fitting since it was formulated to describe the non-linear relationship between stress and strain in materials near their yield points. The equations for the 


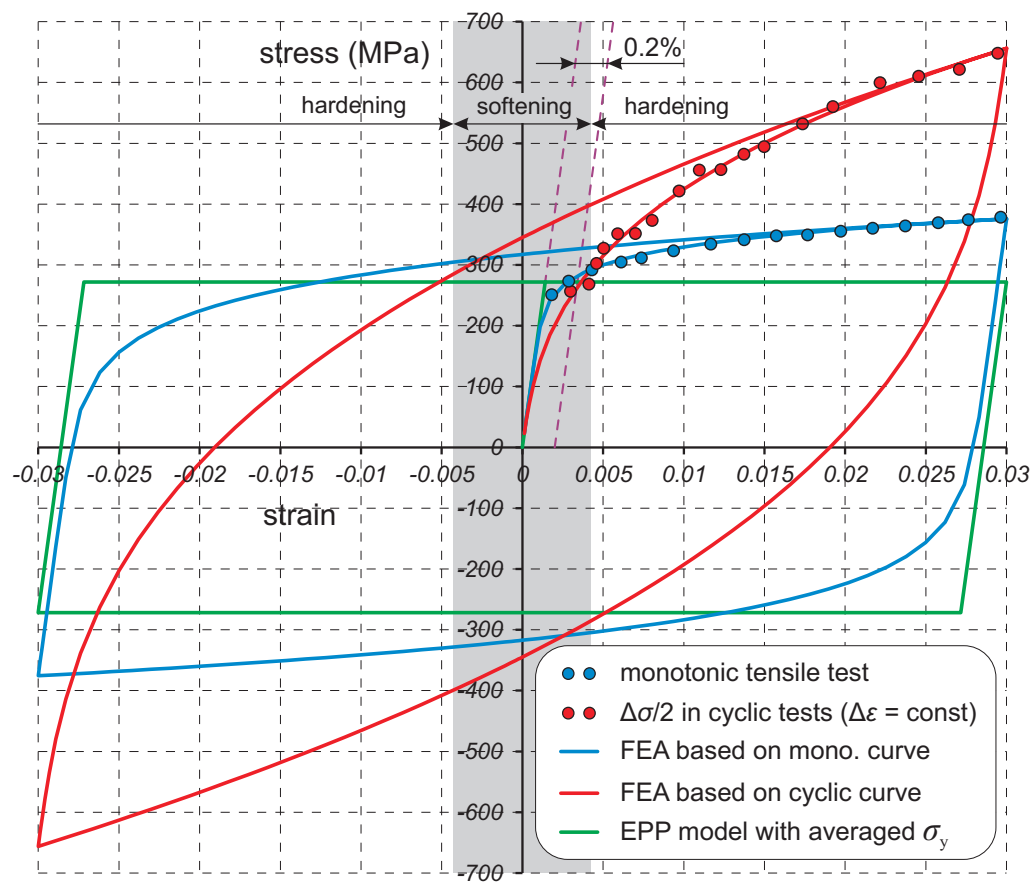

Figure 2: Experimental stress-strain curves [5] of AISI type $316 \mathrm{~N}(\mathrm{~L})$ steel at $20^{\circ} \mathrm{C}$ and fittings with the R-O (1) and MLKH models.

Table 1: Material parameters of AISI type $316 \mathrm{~N}(\mathrm{~L})$ steel at $20^{\circ} \mathrm{C}$ corresponding to the R-O (1) and EPP plasticity models.

\begin{tabular}{l|ll|l}
\hline \hline \multirow{2}{*}{ Type } & \multicolumn{2}{|l|}{ Ramberg-Osgood } & EPP \\
\cline { 2 - 4 } & $B(\mathrm{MPa})$ & $\beta$ & $\sigma_{\mathrm{y}}(\mathrm{MPa})$ \\
\hline Monotonic & 551.18 & 0.1075 & 282.6 \\
Cyclic & 2379.07 & 0.3553 & 261.5 \\
Averaged & - & - & 272.04 \\
\hline \hline
\end{tabular}

with $E=194(\mathrm{GPa})$ and $\nu=0.27$ after [6]. 
monotonic and cyclic SSCs are as follows:

$$
\varepsilon^{\mathrm{tot}}=\frac{\sigma}{E}+\left(\frac{\sigma}{B}\right)^{1 / \beta} \quad \text { and } \quad \frac{\Delta \varepsilon^{\mathrm{tot}}}{2}=\frac{\Delta \sigma}{2 E}+\left(\frac{\Delta \sigma}{2 B}\right)^{1 / \beta},
$$

where $\Delta \varepsilon_{\text {tot }}$ is the total strain range and $\Delta \sigma$ is the total stress range (MPa) for each cyclic test respectively; $B$ and $\beta$ are material constants. The elastic properties used in both R-O and elastic-perfectly-plastic (EPP) models are the Young's modulus $E$ in MPa and the Poisson's ratio $\nu$.

The elastic material properties at $20^{\circ} \mathrm{C}$ are taken from [6] and given in Table 1. Using the defined value of $E$, the total strain $\varepsilon^{\text {tot }}$ in the experimental curves is decomposed into elastic and plastic strain. Then the plastic component $\varepsilon^{\mathrm{p}}$ of strain is fitted using the least squares method by the following relations, which are derived from the Eq. (1):

$$
\sigma=B\left(\varepsilon^{\mathrm{p}}\right)^{\beta} \quad \text { and } \quad \frac{\Delta \sigma}{2}=B\left(\frac{\Delta \varepsilon^{\mathrm{p}}}{2}\right)^{\beta},
$$

where the resultant values of $\mathrm{R}-\mathrm{O}$ material constants $(B$ and $\beta)$ are reported in Table 1. The R-O fits for monotonic and cyclic SSCs are then used to identify the constants for the material models used for FEA.

Since the EPP model is not able to produce hardening, the multilinear kinematic hardening (MLKH) model is applied to describe both types of curves [5]. The results of the MLKH model verification in ANSYS with a single cyclic FEsimulation of a uniaxial specimen at $\Delta \varepsilon_{\text {tot }}=6 \%$ are shown in Fig. 2. It confirms a very good match of the experiments [5] by the MLKH model with two different types of material response.

\section{Advanced structural FEA of the PRV operation}

\subsection{FE-model of the PRV with BCs and loadings}

Since our main focus is an investigation of the structural behaviour only in the contact area, the available 3D CAD-model of the PRV was drastically simplified. The FE-model intended for an analysis is axisymmetric and consists of only 3 components as illustrated in Fig. 3. The most important component, top part of the nozzle, is reproduced in detail, while the representation of the disc and spring is significantly simplified. The spring is compressed by application of the particular vertical displacement $\Delta_{\mathrm{sp}}$ to its top end. This displacement $\Delta_{\mathrm{sp}}$ actuates a particular sealing force, which corresponds to a predefined $P_{\text {set }}$ as explained in the next section. The spring force keeps the disc in balance, when the internal pressure $P$ reaches the corresponding value of $P_{\text {set }}$. The internal pressure $P$ is applied to all interior faces of the nozzle and disc except two lines, which form the contact pair. This contact pair has an activated FPP feature, which allows the change of the pressure conditions automatically according to the contact conditions. In this case it propagates an internal pressure $P$ into the contact gap, 


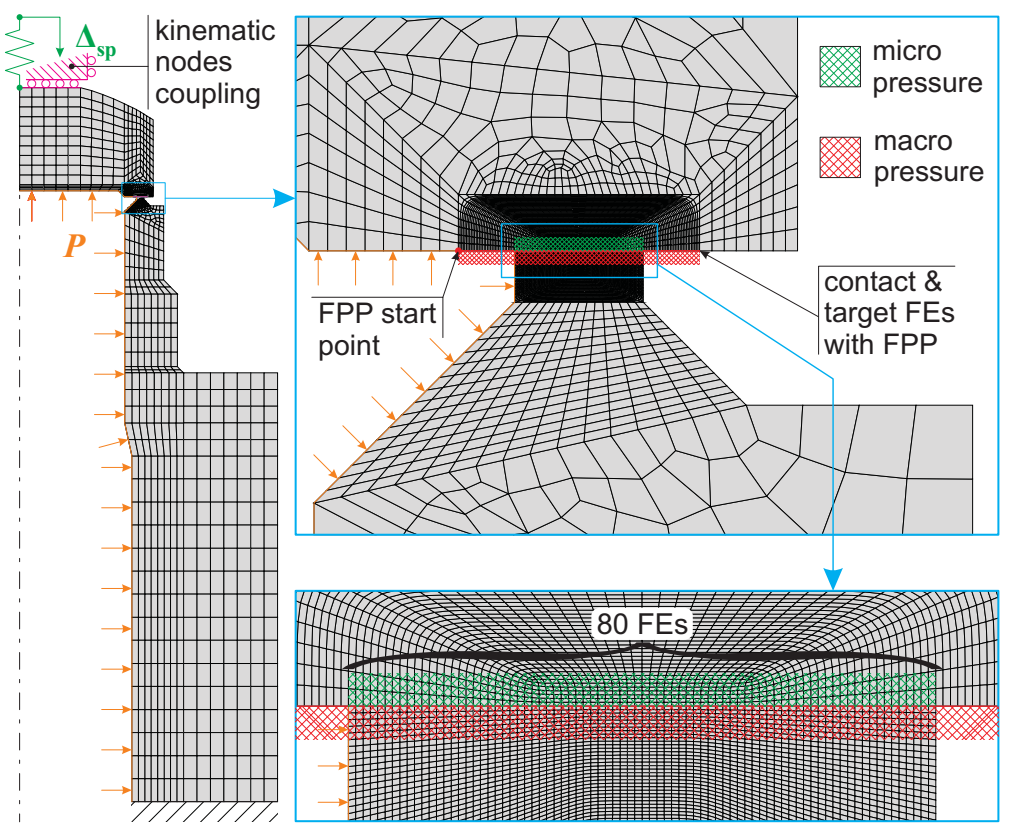

Figure 3: FE-model of the valve with detained view of the contact face, BCs and loadings.

when it opens due to plastic deformation of the internal edge of the contact face. This numerical technique in ANSYS enables a macro-component of the pressure penetration as shown in Fig. 1a. The micro-component is taken into account by the application of a non-uniform pressure distributed over the contact face as shown in Fig. 1b. The actual form of pressure distribution depends on the compressibility of fluid and is defined by the function of pressure dependent on the radial coordinate. The contact area, where the micropressure is applied, is variable and changes in order to avoid an overlap with the macropressure. It should be noted that 80 solid and contact FEs are created over the contact face in order to obtain a highresolution representation of the pressure profile before the valve lift as shown in Fig. 1c.

\subsection{Pressure profiles and sealing force}

The microscopic component of the FPP is applied to the contact face using an analytical function, which needs to be defined in ANSYS as dependent on internal pressure, geometric parameters and type of fluid. Müller and Nau [9] provided a general analytical expression for a pressure drop in a uniform annular seal gap 
filled with a fluid as follows:

$$
p(x)=p_{1}\left[1-\left(1-\gamma^{2}\right) \frac{x}{L}\right]^{n},
$$

where $L$ - length of a seal gap; $p_{1}$ - internal pressure and $p_{2}$ - external pressure; $\gamma=p_{2} / p_{1}$ - pressure ratio and $n$ - power-law exponent, which is dependent on the type of fluid $-n=0.5$ for gas and $n=1$ for liquid.

Equation (3) may be extended to the case of a plane contact gap in the valve. Since the profile of microscopic pressure distribution remains the same, the mathematical form of pressure drop in the seal gap before valve opening is slightly changed to

$$
P(r)=P_{\text {set }}\left[\frac{r_{\text {out }}-r}{r_{\text {out }}-r_{\text {fpp }}}\right]^{n},
$$

where $P_{\text {set }}$ - set pressure corresponding to the balance of forces applied to the disk, $r_{\text {out }}$ - outer radius of the contact area, $r_{\text {in }}$ - inner radius of the contact area or radius of the orifice, $r_{\mathrm{fpp}}$ - radius of fluid pressure penetration (FPP). It should be noted that $\left(r_{\text {out }}<r_{\mathrm{fpp}} \leq r_{\mathrm{in}}\right)$ if FPP is available, and $r_{\mathrm{fpp}}=r_{\mathrm{in}}$ if FPP is unavailable.

Integrating Eq. (4) by $r$ over the length of the valve seat $\left(L_{\text {seat }}=r_{\text {out }}-r_{\text {in }}\right)$ from $r_{\text {in }}$ to $r_{\text {out }}$, an average value of the pressure within the pressure profile is obtained in analytical form:

$$
\bar{P}=\frac{P_{\text {set }}}{1+n} .
$$

Based upon the proposed concept, the total force, which needs to be actuated in the spring during its preload in order to lift the valve at a set pressure $P_{\text {set }}$, consists of the three components:

1. Orifice force or force produced by pressure $P_{\text {set }}$ acting on the surface of the disc corresponding to the area of the orifice:

$$
F_{\text {or }}=P_{\text {set }} \pi r_{\text {in }}^{2}
$$

2. Macro-fluid force, which is calculated assuming a stepped distribution of pressure in the area of the macroscopic FPP:

$$
F_{\mathrm{ma}}=P_{\mathrm{set}} \pi\left(r_{\mathrm{fpp}}^{2}-r_{\mathrm{in}}^{2}\right)
$$

3. Micro-fluid force, which is calculated using the average pressure (5) over the area of the microscopic FPP:

$$
F_{\mathrm{mi}}=\bar{P} \pi\left(r_{\mathrm{out}}^{2}-r_{\mathrm{fpp}}^{2}\right)
$$

Thus, the total spring force includes an additional 'seat' component as:

$$
F_{\text {tot }}=F_{\text {or }}+F_{\text {seat }}, \quad \text { where } \quad F_{\text {seat }}=F_{\text {ma }}+F_{\text {mi }} .
$$




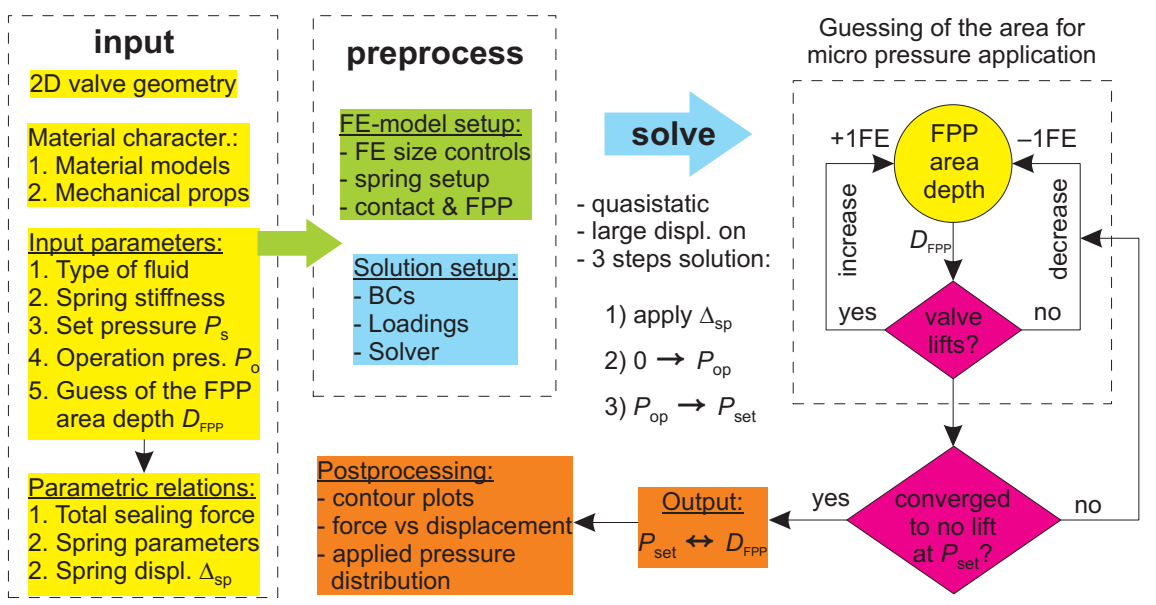

Figure 4: Flowchart of the iterative FEA to predict the pressure distribution over the contact area.

\subsection{FE-analysis setup and solution}

An advanced FE-analysis in this study was developed and automated using APDLscript. It enables essential flexibility and makes this study parametric since all important parameters can be changed and adjusted. The analysis flow consists of several steps as illustrated in Fig. 4:

1. Preliminary operations including parametric calculations.

2. Preprocessing including FE-model and solution setup.

3. Solution including solver setup and running solution.

The key analysis procedure is an iterative guessing of the depth $D_{\mathrm{fpp}}$ of the macroscopic FPP, which is expressed in terms of the number of FEs $N_{\text {fpp }}$ along the contact face from the internal edge up to the boundary of macroscopic FPP as shown in Fig. 1. Since the horizontal dimension of each FE along the contact face is $D_{\mathrm{FE}}=1.25 / 80=0.015625 \mathrm{~mm}$ (see Fig. 3), the output parameter is defined as $D_{\mathrm{fpp}}=N_{\mathrm{fpp}} \cdot D_{\mathrm{FE}}$. The objective of this process is a fitting of microscopic FPP described by the function (4) to actual macroscopic FPP, which is obtained from the FEA results. This procedure needs to be implemented since the degree of contact face global deformation, which defines the macroscopic FPP, is unknown for each combination of loading. In other words, this procedure is a manual coupling of the macro-component of the FPP with the micro-component by variation of $r_{\mathrm{fpp}}=r_{\mathrm{in}}+D_{\mathrm{fpp}}$ in the function (4). When correctly guessing the value of $r_{\mathrm{fpp}}$, the valve lifts exactly at $P_{\text {set }}$ without a gap or overlapping of the macro- and micro-components of FPP. As shown in Fig. 4, in the case of a gap (when the valve doesn't lift), $D_{\mathrm{fpp}}$ is decreased by $1 \mathrm{FE}$ length $D_{\mathrm{FE}}$, while in the case of an overlap (when the valve lifts too early), $D_{\text {fpp }}$ is increased by $1 \mathrm{FE}$ length $D_{\mathrm{FE}}$. Once this iterative procedure has converged to no lift of the valve 

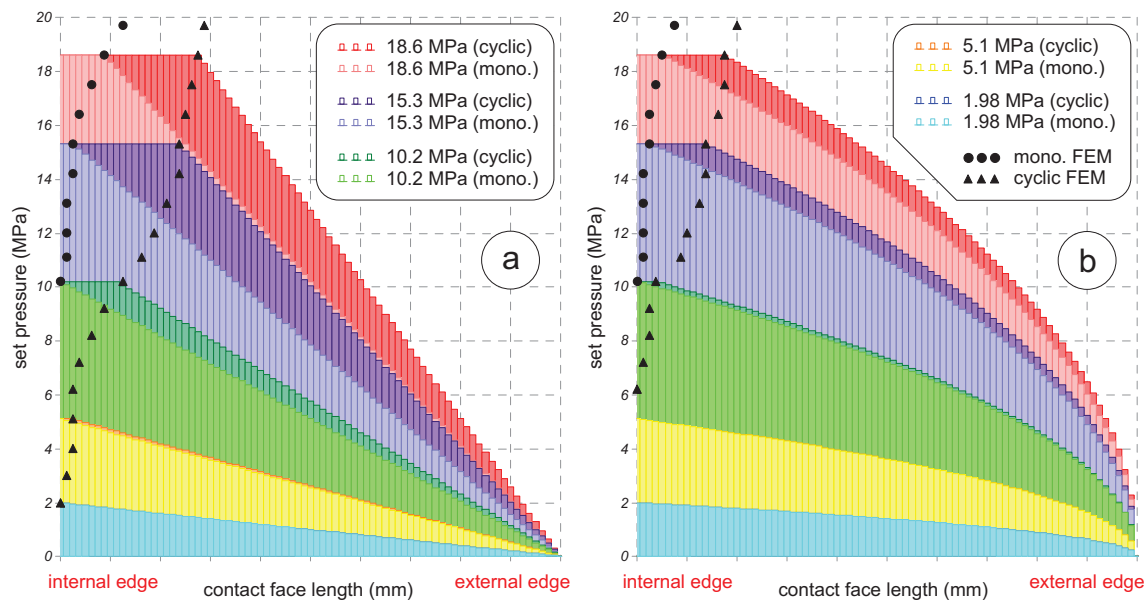

Figure 5: FEA results with FPP and fluid pressure drop in contact area at different set pressures for (a) liquid and (b) gas - markers denote the boundaries macroscopic and microscopic FPP.

when $P \leq P_{\text {set }}$, the main results are output in the form of values pair ( $P_{\text {set }}$ and corresponding $\left.D_{\mathrm{fpp}}\right)$.

\section{Discussion and conclusions}

The FEA have been performed for the wide range of $P_{\text {set }}$ comprising 21 values (1.98-23.0 MPa). Each simulation was done for two different types of fluid (liquid or gas) and two different types of material response (monotonic or cyclic). These make up a total of 84 FE-simulations, which were manually controlled to give a converged pressure profile on the contact face. Each variant of FEA required at least 5 attempts to achieve a converged result. Therefore, about 500 FE-simulations have been performed in this study.

Obtained results are shown in Fig. 5 in the form of dots and triangles for all values of $P_{\text {set }}$ and in the form of pressure profiles over the contact face for 5 standard values of $P_{\text {set }}$. The degree of macroscopic FPP increases non-linearly with an increase of $P_{\text {set }}$ for both types of fluids. However, the particular depth of global FPP $D_{\mathrm{fpp}}$ and corresponding length of effective contact area $\left(L_{\mathrm{eff}}=\right.$ $L_{\text {seat }}-D_{\text {fpp }}$ ) is quite different for liquid and gas. The other important effect should also be noted that the effective contact length $L_{\text {eff }}$ drastically decreases with cyclic operation of the valve.

The numerical results were fitted by analytical functions, which were used for the formulation of relations for additional 'seat' component of the spring force. The number of finite elements (FEs) involved in FPP at the macroscale is fitted by a smoothing function. It is dependent on $P_{\text {set }}$ and uses an approximation of the 

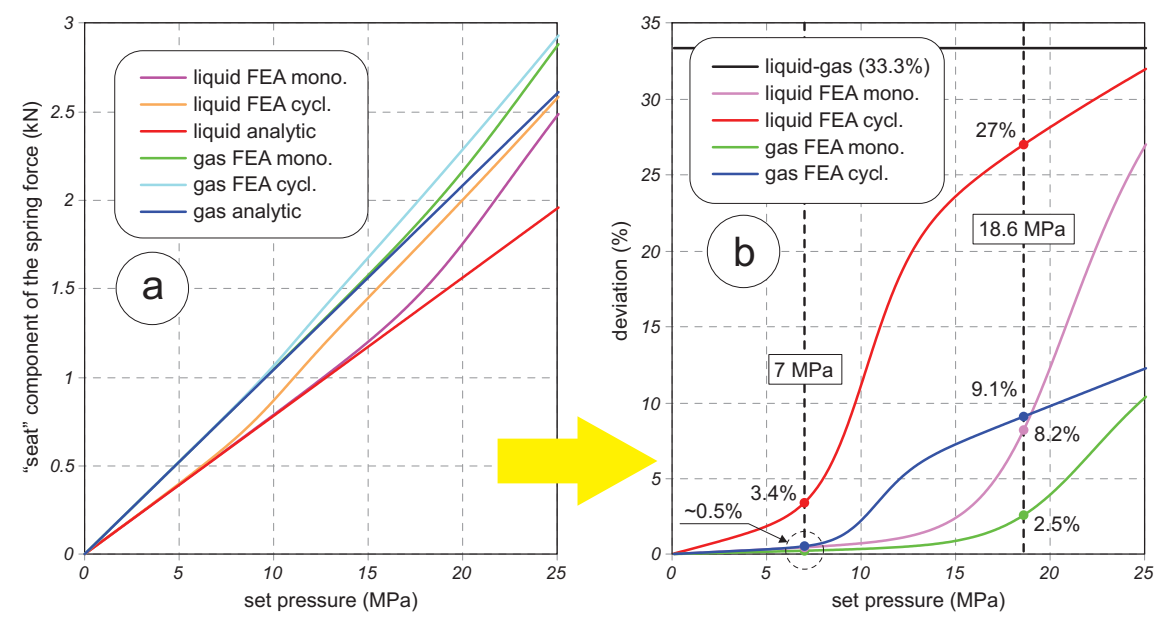

Figure 6: Additional 'seat' component of the spring force: (a) comparison of the formulations defined using FEA and analytically, (b) deviation of FEA predictions from analytical $(\%)$.

Heaviside step function $H\left(P_{\text {set }}\right)$ :

$$
N_{\text {fpp }}\left(P_{\text {set }}\right)=F_{\text {hi }}\left(P_{\text {set }}\right) H\left(P_{\text {set }}\right)+F_{\text {lo }}\left(P_{\text {set }}\right)\left[1-H\left(P_{\text {set }}\right)\right],
$$

where the specific formulations of the step function $H\left(P_{\text {set }}\right)$ and fitting functions for the low pressure $F_{\text {lo }}\left(P_{\text {set }}\right)$ and high pressure $F_{\text {hi }}\left(P_{\text {set }}\right)$ domains are different for 4 combinations of fluid type and material response.

The number of FEs in FPP at the macroscale (10) is then transformed into the function for corresponding radius of FPP as

$$
r_{\text {fpp }}\left(P_{\text {set }}\right)=r_{\text {in }}+\frac{L_{\text {seat }}}{80} N_{\text {fpp }}\left(P_{\text {set }}\right)
$$

Using the value of $r_{\mathrm{fpp}}$ defined by Eq. (11), an additional 'seat' component of the spring force is obtained from Eqs (7)-(9):

$$
F_{\text {seat }}\left(P_{\text {set }}\right)=P_{\text {set }} \pi\left(\left[r_{\text {fpp }}^{2}\left(P_{\text {set }}\right)-r_{\text {in }}^{2}\right]+\frac{r_{\text {out }}^{2}-r_{\text {fpp }}^{2}\left(P_{\text {set }}\right)}{1+n}\right) .
$$

The analytical estimation is done considering microscopic FPP only, i.e. using Eq. (8) for the whole seat contact area. The FEA-based estimation is done considering a micro-macro interaction and corresponding Eq. (12) based on numerical results fitted by the function (10). The deviation of FEA-based force from analytical predictions is much more significant for liquid than for gas, and it increases with increase of $P_{\text {set }}$, as shown in Fig. 6. For example, in the case of $P_{\text {set }}=186$ bar, liquid and cyclic material response, the difference between additional spring forces is about $27 \%$. 
Referring to [3], metal-seated spring valves with operating pressures 90-95\% of $P_{\text {set }}$ do not stay tight for long and usually get damaged after a couple of operations. This fact was confirmed by the advanced FEA implemented in this study using monotonic and cyclic plastic response. The effective contact area of the valve seat changes significantly during the cyclic operation of the valve. In this regard, a spring force required to provide a leakage tightness of the valve needs to be adjusted correspondingly after each resetting. The results of the analysis demonstrate that an alteration of the spring force during cyclic operation may be over a quarter of its initial value. Analyses of the macro deformation of the valve seat/disc under various pressures using quasistatic structural FEA with FPP technique revealed that:

1. Macro deformation is important and affects sealing area.

2. Cyclic material response affects the structural behaviour.

3. The type of fluid influences the contact pressure distribution.

\section{References}

[1] Malek, M.A., Pressure Relief Devices: ASME and API Code Simplified. McGraw-Hill: New York, USA, 2006.

[2] Song, X.-G., Park, Y.-C. \& Park, J.-H., Blowdown prediction of a conventional pressure relief valve with a simplified dynamic model. Math \& Comp Modelling, 57(1-2), pp. 279-288, 2013.

[3] Hellemans, M., The Safety Relief Valve Handbook: Design and Use of Process Safety Valves to ASME and International Codes and Standards. ButterworthHeinemann: Oxford, UK, 2009.

[4] Gorash, Y., Altenbach, H. \& Lvov, G., Modelling of high-temperature inelastic behaviour of the austenitic steel AISI type 316 using a continuum damage mechanics approach. Journal of Strain Analysis, 47(4), pp. 229-243, 2012.

[5] Chaboche, J.-L., Dang Van, K. \& Cordier, G., Modelization of the strain memory effect on the cyclic hardening of 316 stainless steel. Trans. 5th Int. Conf. on Structural Mechanics in Reactor Technology, IASMiRT: Berlin, Germany, number L11/3 in SMiRT5, pp. 1-10, 1979.

[6] Karditsas, P.J. \& Baptiste, M.-J., Thermal and structural properties of fusion related materials. ARIES Properties Archive: UKAEA FUS 294, Euratom/UKAEA Fusion Association, San Diego, 1995.

[7] Nouailhas, D., Cailletaud, G., Policella, H., Marquis, D., Dufailly, J., Lieurade, H.P., Ribes, A. \& Bollinger, E., On the description of cyclic hardening and initial cold working. Engineering Fracture Mechanics, 21(4), pp. 887-895, 1985.

[8] Ramberg, W. \& Osgood, W.R., Description of stress-strain curves by three parameters. Technical Note no. 902, National Advisory Committee For Aeronautics, NASA, Washington DC, USA, 1943.

[9] Müller, H.K. \& Nau, B.S., Fluid Sealing Technology: Principles and Applications. Marcel Dekker, Inc.: New York, USA, 1998. 